Tirage à part de la

REVUE SUISSE D'HYDROLOGIE

VERIAG BIRKHÄUSR BASEI.

Vol. XIII $1951 \quad$ Fasc. 2

La «Matrix》 des Poissons

VI.

\title{
Etudes oscillographiques sur la détermination du caractère nocif de différentes eaux usées de provenance industrielle ${ }^{1}$ )
}

\author{
Par Ulrich A. CORTI
}

Manuscrit reçu le I2 avril I95I

Institut fédéral pour l'aménagement, l'épuration et la protection des eaux, annexé à l'Ecole polytechnique fédérale, Zurich

Par la construction d'une installation, basée sur des principes sismiques $[\mathrm{I}]^{2}$ ), et permettant d'enregistrer par oscillographie les vibrations ou mouvements d'organismes aquicoles transvasés dans un bassin d'eau suspendu, il nous fut possible d'étudier, par une méthode nouvelle, le comportement de poissons sous l'influence de matières toxiques.

Pour les essais en question, des truites arc-en-ciel, Salmo irideus W. Gibb., furent soumises à l'action des combinaisons chimiques suivantes:

$\begin{array}{ll}\text { phénol } & \alpha \text {-naphtol } \\ \text { pyrocatéchine } & \beta \text {-naphtol } \\ \text { résorcine } & \text { vanilline } \\ \text { hydrochinone } & \text { aldéhyde vératrique } \\ \text { pyrogallol } & \text { héliotropine (pipéronal) } \\ \text { phloroglucine } & \end{array}$

Il s'agit donc, en partie, de produits de la grande industrie chimique. Du moins des traces des substances mentionnées se trouvent presque toujours dans les eaux résiduaires des usines respectives.

1) Communication présentée au XXII Congrès de Chimie industrielle, le 27 octobre 1949, à Barcelone. Vu l'impossibilité de recevoir des indications précises sur la date de la publication des comptes rendus du dit congrès, l'auteur s'est décidé de publier cette étude dans cette Revue. La $5^{\circ}$ communication a paru dans les Comptes rendus de la Société helvétique des Sciences naturelles, p. 159 à 161 (Lausanne 1949).

2) Voir Références à la page 364 . 
Tableau 1

\begin{tabular}{|c|c|c|c|c|c|c|c|c|c|}
\hline \multirow{2}{*}{$\mathrm{N}^{\circ}$} & \multicolumn{3}{|c|}{ Truite } & \multirow{2}{*}{$\begin{array}{c}\text { Substance } \\
\text { mg/1 } \\
\text { (eau du robinet) }\end{array}$} & \multirow{2}{*}{$\mathrm{pH}$} & \multirow{2}{*}{$\underset{\substack{\text { Temp. } \\
{ }^{\circ} \mathrm{C}}}{ }$} & \multirow{2}{*}{$\begin{array}{c}\text { Première } \\
\text { réaction } \\
\text { après }\end{array}$} & \multirow{2}{*}{$\begin{array}{c}\text { Poisson } \\
\text { sur le } \\
\text { dos après }\end{array}$} & \multirow{2}{*}{$\begin{array}{c}\text { Dernière } \\
\text { réaction } \\
\text { après }\end{array}$} \\
\hline & $\begin{array}{l}\text { poids } \\
\mathrm{g}\end{array}$ & $\begin{array}{c}\text { âge } \\
\text { jours }\end{array}$ & sexe & & & & & & \\
\hline 1 & 98,8 & 685 & f & $\begin{array}{l}\text { phénol, } \\
15,7 \mathrm{mg}\end{array}$ & 7,80 & 12 & $1^{\prime} 20^{\prime \prime}$ & $4^{\prime} 17^{\prime \prime}$ & $4^{\prime} 45^{\prime \prime}$ \\
\hline 2 & 112,0 & 687 & $f$ & $\begin{array}{l}\text { phénol, } \\
15,7 \mathrm{mg}\end{array}$ & 7,80 & 11. & $1^{\prime} 10^{\prime \prime}$ & $3^{\prime} 30^{\prime \prime}$ & $4^{\prime} 15^{\prime \prime}$ \\
\hline 3 & 84,3 & 682 & i & $\begin{array}{l}\text { phénol, } \\
31,3 \mathrm{mg}\end{array}$ & 7,81 & 12 & $30^{\prime \prime}$ & $1^{\prime} 37^{\prime \prime}$ & $4^{\prime} 39^{\prime \prime}$ \\
\hline 4 & $1.19,0$ & 682 & $\mathrm{~m}$ & $\begin{array}{l}\text { phénol, } \\
31,3 \mathrm{mg}\end{array}$ & 7,81 & 12 & $35^{\prime \prime}$ & $1^{\prime} 33^{\prime \prime}$ & $4^{\prime} 51^{\prime \prime}$ \\
\hline 5 & 118,4 & 678 & $m$ & $\begin{array}{l}\text { pyrocatéchine, } \\
18,3 \mathrm{mg}\end{array}$ & 7,69 & 12 & $4^{\prime}+9^{\prime \prime}$ & $8^{\prime} 12^{\prime \prime}$ & $10^{\prime} 01^{\prime \prime}$ \\
\hline 6 & 156,4 & 678 & f & $\begin{array}{l}\text { pyrocatéchine, } \\
18,3 \mathrm{mg}\end{array}$ & 7,69 & 11. & $6^{\prime} 58^{\prime \prime}$ & $12^{\prime} 01^{\prime \prime}$ & $13^{\prime} 31^{\prime \prime}$ \\
\hline 7 & 167,7 & 673 & $\mathrm{f}$ & $\begin{array}{l}\text { pyrocatéchine, } \\
36,7 \mathrm{mg}\end{array}$ & 7,72 & 11 & $2^{\prime} 10^{\prime \prime}$ & $4^{\prime} 16^{\prime \prime}$ & $6^{\prime} 27^{\prime \prime}$ \\
\hline 8 & 131,5 & 672 & $f^{\prime}$ & $\begin{array}{l}\text { pyrocatéchine, } \\
36,7 \mathrm{mg}\end{array}$ & 7,72 & 12 & $1^{\prime} 02^{\prime \prime}$ & $\longrightarrow$ & $4^{\prime} 06^{\prime \prime}$ \\
\hline 9 & 144,4 & 673 & $\mathrm{~m}$ & $\begin{array}{l}\text { pyrocatéchine, } \\
36,7 \mathrm{mg}\end{array}$ & 7,72 & 12 & $1^{\prime} 52^{\prime \prime}$ & $4^{\prime} 08^{\prime \prime}$ & $5^{\prime} 34^{\prime \prime}$ \\
\hline 10 & 161,0 & 676 & f & $\begin{array}{l}\text { résorcine, } \\
36,7 \mathrm{mg}\end{array}$ & 7,73 & 11 & $1^{\prime} 21^{\prime \prime}$ & $\cdots$ & - \\
\hline 11 & 86,8 & 680 & f & $\begin{array}{l}\text { hydrochinone, } \\
36,7 \mathrm{mg}\end{array}$ & 7,78 & 12 & $3^{\prime} 11^{\prime \prime}$ & -- & $\cdots$ \\
\hline 12 & 146,9 & 679 & $\mathrm{~m}$ & $\begin{array}{l}\text { pyrogallol, } \\
38,0 \mathrm{mg}\end{array}$ & 7,42 & 12 & $1^{\prime} 09^{\prime \prime}$ & $\cdots$ & $\cdots$ \\
\hline 13 & 116,7 & 679 & $\mathrm{f}$ & $\begin{array}{l}\text { phloroglucine, } \\
42,0 \mathrm{mg}\end{array}$ & 7,80 & 12 & $1^{\prime} 04^{\prime \prime}$ & -- & $\cdots \cdots-$ \\
\hline 14 & 103,0 & 679 & $\mathrm{f}$ & $\begin{array}{l}\alpha \text {-naphtol, } \\
48,0 \mathrm{mg}\end{array}$ & 7,76 & 11 & $1^{\prime} 18^{\prime \prime}$ & $2^{\prime} 51^{\prime \prime}$ & $6^{\prime} 06^{\prime \prime}$ \\
\hline 15 & 100,1 & 681 & $\mathbf{f}$ & $\begin{array}{l}\alpha \text {-naphtol, } \\
48,0 \mathrm{mg}\end{array}$ & 7,76 & 11. & $45^{\prime \prime}$ & $2^{\prime} 08^{\prime \prime}$ & $5^{\prime} 34^{\prime \prime}$ \\
\hline 16 & 88,4 & 681 & $\mathrm{~m}$ & $\begin{array}{l}\beta \text {-naphtol, } \\
48,0 \mathrm{mg}\end{array}$ & 7,92 & 11 & $21^{\prime \prime}$ & $1^{\prime} 28^{\prime \prime}$ & $6^{\prime} 56^{\prime \prime}$ \\
\hline 17 & 115,3 & 680 & $\mathrm{f}$ & $\begin{array}{l}\beta \text {-naphtol, } \\
48,0 \mathrm{mg}\end{array}$ & 7,92 & 11 & $1^{\prime} 11^{\prime \prime}$ & $2^{\prime} 09^{\prime \prime}$ & $6^{\prime} 43^{\prime \prime}$ \\
\hline 18 & 124,7 & 622 & $\mathrm{~m}$ & $\begin{array}{l}\text { vanilline, } \\
56,7 \mathrm{mg}\end{array}$ & 7,4 & 11 & - & - & - \\
\hline 19 & 121,4 & 623 & $\mathrm{~m}$ & $\begin{array}{l}\text { aldéhyde vératrique } \\
55,3 \mathrm{mg}\end{array}$ & 7,6 & 11 & $\cdots$ & $\therefore$ & - \\
\hline 20 & 121,8 & 623 & $\mathrm{~m}$ & $\begin{array}{l}\text { héliotropine, } \\
50,0 \mathrm{mg}\end{array}$ & 7,7 & 11 & $2^{\prime} 44^{\prime \prime}$ & -- & $15^{\prime} 00^{\prime \prime}$ \\
\hline
\end{tabular}


Les concentrations choisies des solutions varient pour nos essais de $\sim \mathrm{I}, 67 \cdot \mathrm{IO}^{-4}$ à $\sim 3,33 \cdot \mathrm{IO}^{-4}$ moles/litre, la température de l'eau du bassin de II à $12^{\circ} \mathrm{C}$, le pH de 7 à 8 environ, le poids des truites de 84 à $\mathrm{I} 68 \mathrm{~g}$ (mâles et femelles) et leur âge de 622 à 7I 5 jours (comptés à partir du jour de l'éclosion des poissons). Les doses, un peu massives, des combinaisons chimiques permirent de constater l'effet toxique aigu d'une façon très caractéristique. Nous avons l'intention de diminuer encore la concentration

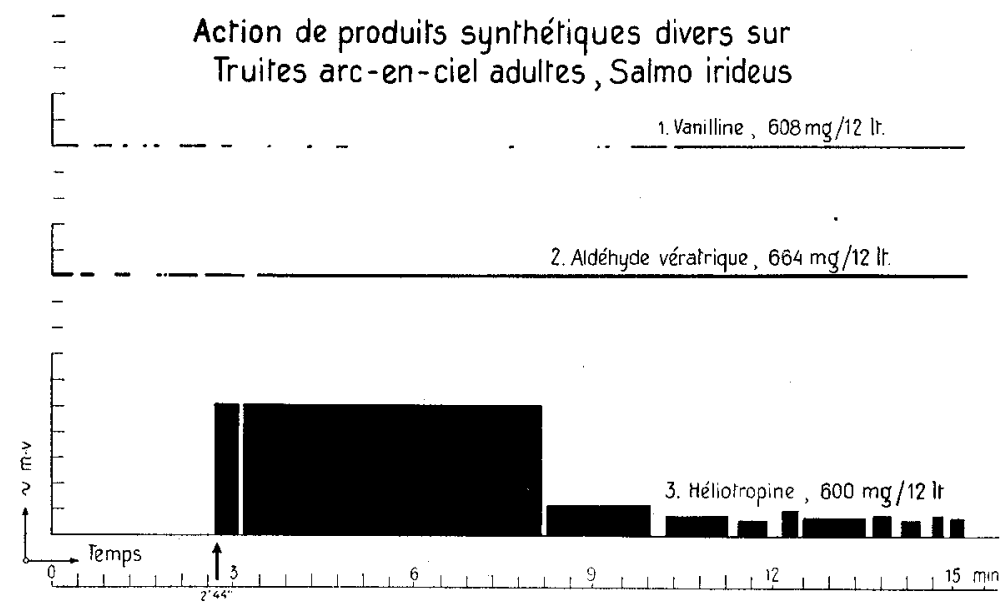

Figure 1.

choisie, afin de déterminer la dilution minimale où l'intoxication se manifeste encore d'une manière nette.

Pour chaque essai (une seule truite), le moment de Pintroduction du poisson dans le milieu environnant à caractériser, celui de la première réaction (choc) de la truite, le moment où l'individu se tourne sur le dos et la fin des mouvements du poisson furent marqués. Ainsi nous avons obtenu les résultats indiqués au tableau I.

Il résulte de ces chiffres que:

$I^{\circ}$ Les truites arc-en-ciel réagissent bien comme «bioindicateurs».

$2^{\circ}$ L'action toxique aiguë des combinaisons «phénoliques» appliquées est très forte pour: phénol, pyrocatéchine, $\alpha$ - et $\beta$-naphtol et héliotropine, mais relativement faible pour: résorcine, hydrochinone, phloroglucine, pyrogallol, vanilline et aldéhyde vératrique. 
$3^{\circ}$ La toxicité de produits chimiques de constitution très semblable peut être très différente. Un cas tout à fait étonnant de ce genre est représenté par la triade vanilline, aldéhyde vératrique et héliotropine:<smiles>COC1CC(C=O)CCC1O</smiles>

vanilline (peu toxique)<smiles>COC1CCC(C=O)CC1OC</smiles>

aldéhyde vératrique (peu toxique)<smiles>O=CC1CCC2OCOC2C1</smiles>

héliotropine (pipéronal) (très toxique)

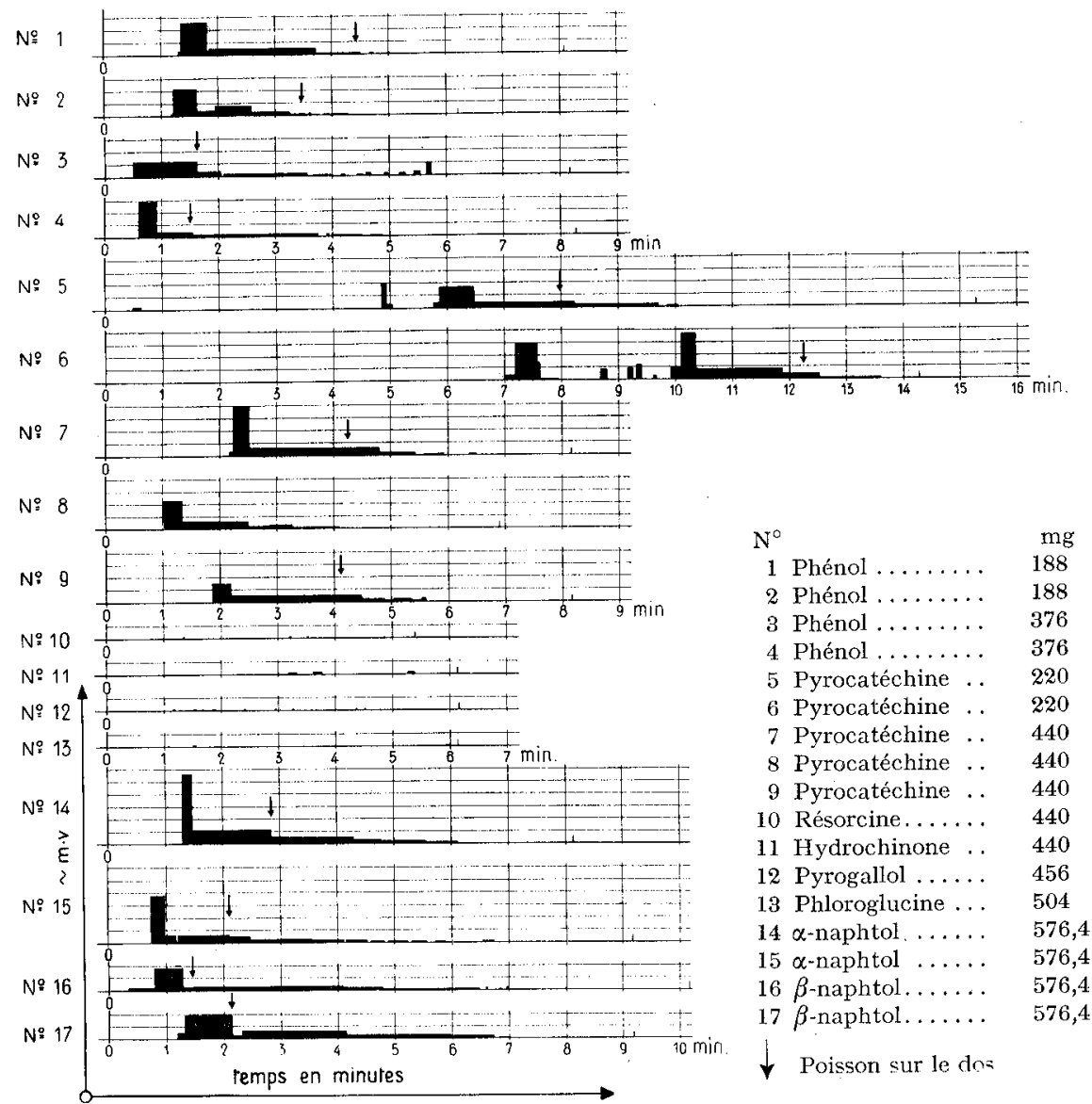

Fign? 
Il semble donc qu'on peut arriver par notre méthode à caractériser d'une façon aussi élégante que rapide le degré de toxicité de substances dont la constitution ne varie que très peu, surtout si l'on tient compte du fait que le bassin peut être chargé sans autre d'une douzaine de poissons à la fois, afin d'obtenir d'un coup des résultats statistiques.

L'image typique des empoisonnements ressort non seulement des oscillogrammes originaux, mais aussi des graphiques que l'on peut en déduire

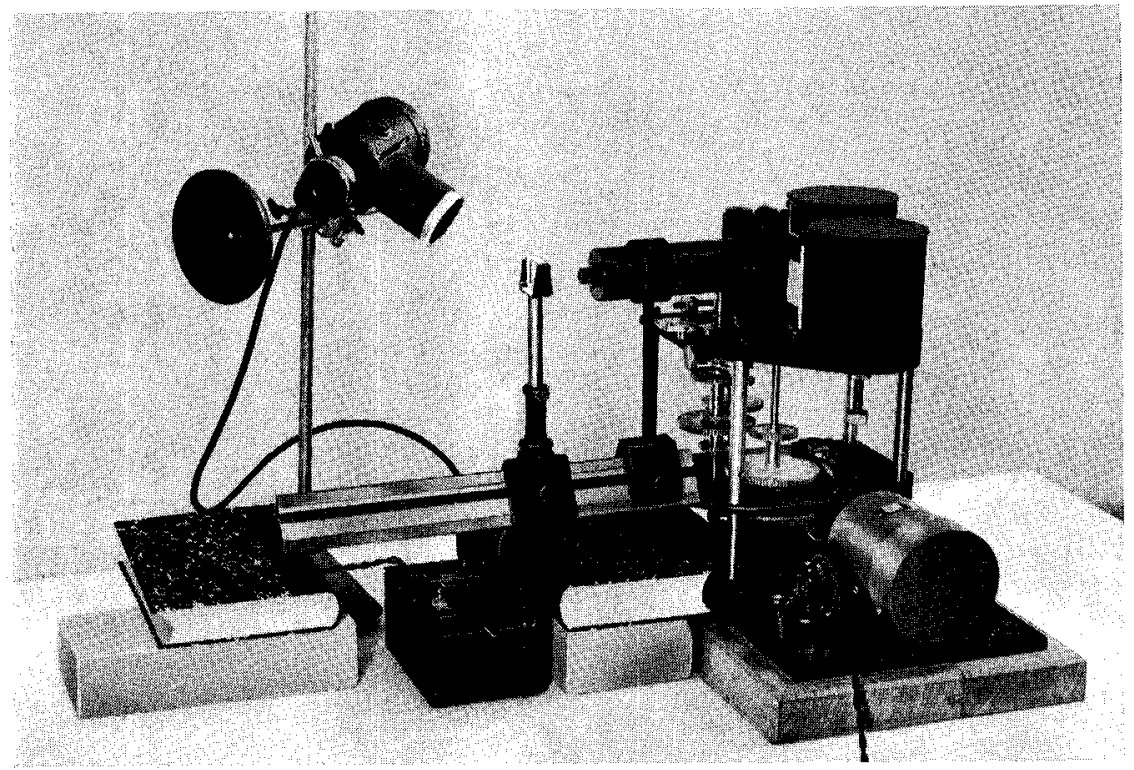

Figure 3

Dispositif pour l'enregistrement des mouvements de petits organismes. A gauche la source de lumière, au centre la cuvette, contenant les microorganismes, à droite le transporteur du film oscillographique. L'ensemble est placé dans une chambre obscure.

facilement (voir la figure 2), où le temps est indiqué en abscisse et où la hauteur des ordonnées est proportionnelle à $m p(m=$ poids du bassin entier y compris le poisson; $p=$ vitesse du bassin mis en mouvement par la truite). Vu que $m$ est constant pour chaque essai, la division des valeurs indiquées par les ordonnées fournit immédiatement le rapport effectif des vitesses du poisson.

Notre méthode, d'ailleurs très sensible, fut appliquée aussi à un cas pratique. Dans un ruisseau suisse, une quantité considérable de poissons morts 
furent trouvés. On estima que l'égout d'une usine en était la cause. Des échantillons d'eau prélevés en amont et en aval du ruisseau et à l'égout même, mis dans notre bassin avec des poissons, démontrèrent, au bout de très peu de temps, que le déversement de matières toxiques par l'égout était responsable de l'accident en question. Une analyse chimique a confirmé un pourcentage très élevé en $\mathrm{H}_{2} \mathrm{~S}$. L'avantage de notre méthode consiste en ceci, qu'on obtient par les oscillogrammes tout de suite des documents authentiques et absolument objectifs.

En poursuivant les essais décrits précédemment, nous sommes arrivés à travailler avec des micro- ou semi-microorganismes, comme par exemple des Daphnia ou des Tubifex (nourriture des poissons!). Ici la méthode est un peu différente. Nous remplissons des cuvettes en verre, montées sur un banc optique, avec de l'eau à analyser, en projetant la cuvette sur un transporteur de papier (film) oscillographique. Ensuite, un ou plusieurs individus de l'espèce sont introduits soigneusement dans la cuvette au moyen d'une pipette ou d'un fil en platine et l'on photographie le comportement des organismes dans la solution, en utilisant un champ sur fond noir. Ainsi l'on obtient des documents nets des événements qui se passent dans la cuvette.

Nous indiquons ci-après les résultats obtenus avec Tubifex.

Tableau 2

Equivalence toxiqua en grammes et en moles/litre d'eau distillée/30 secondes environ

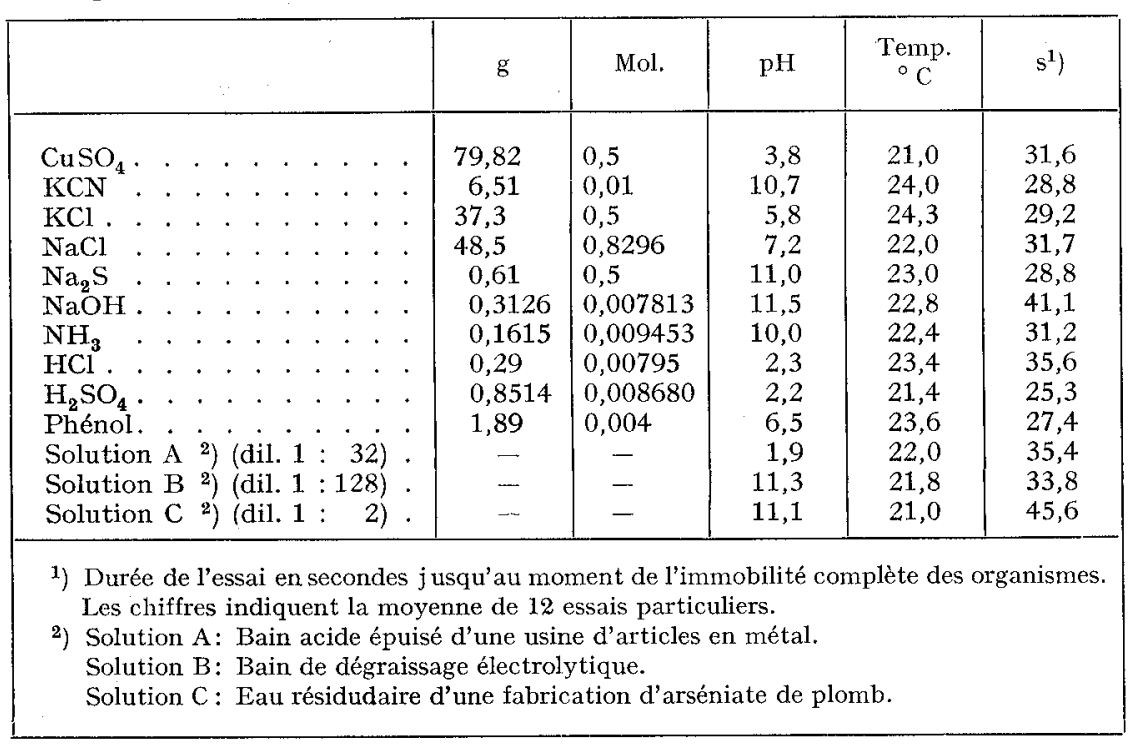


Par conséquent la solution A originale, comme eau résiduaire industrielle, est environ 32 fois plus toxique qu'une solution aqueuse, contenant $\mathrm{I}, 89 \mathrm{~g}$ de phénol/litre. La toxicité de la solution B originale est I 28 fois plus forte que celle d'une solution qui contient 79,82 $\mathrm{g} \mathrm{CuSO}_{4}$ ou 6,5I I g KCN/litre d'eau distillée.

\section{ZUSAMMENFASSUNG}

Oszillograpbische Studien zur Ermittlung der toxischen Natur perschiedener industrieller Abpö̈sser

Ein Aquarium, das mittels vier Drahtlitzen an der Decke des Laboratoriums aufgehängt worden war, wurde mit Wasser, das fischtoxische Eigenschaften aufwies, gefüllt und hierauf pro Versuch ein normaler Fisch (z. B. eine Regenbogenforelle) in das Bassin eingesetzt. Die durch Vergiftung auftretenden Erregungszustände des Fisches werden auf das Aquarium übertragen und die Erschütterungen des letzteren mit seismischen Methoden gemessen und auf einem Film oszillographisch registriert. Die ersten, mit verdünnten, wäßrigen Lösungen von Phenol und einigen seiner Homologen sowie mit drei Aldehyden der aromatischen Serie und industriellem Abwasser erhaltenen Prüfungsergebnisse erweisen die Einfachheit, Eleganz und Brauchbarkeit der neuen Methode. Für Mikroorganismen wurde ein anderes Verfahren entwickelt. Eine Glasküvette, welche das auf toxische Eigenschaften zu prüfende Wasser und die Testorganismen enthält, wird auf einen Oszillographenfilm projiziert und im Dunkelfeld abgebildet. Auf diese Weise erhält man ebenfalls authentische und objektive Dokumente betreffend das Verhalten der Organismen.

\section{RÉFÉRENCES}

[I] CORTI, U.A., et WEBER, M., Untersucbungen über die Vitalität pon Fiscben, Schweiz. Z. Hydrol. 12, 9-I6 (1949).

[2] GRINDLEY, J., Toxicité de quelques substances connues d'être présentes dans les eaux résiduaires déversées dans des ruisseaux, pour la truite arc-en-ciel et le vairon, Ann. appl. Biol. 33, IO3-II2 (I946).

[3] Southgate, B. A., Treatment and Disposal of Industrial Waste Waters, His Majesty's Stationery Office (Londres I948). 